PROCEEDINGS OF THE

AMERICAN MATHEMATICAL SOCIETY

Volume 128, Number 1, Pages 173-182

S 0002-9939(99)05044-3

Article electronically published on June 17, 1999

\title{
NONCONTINUITY OF SPECTRUM FOR THE ADJOINT OF AN OPERATOR
}

\author{
LAURA BURLANDO
}

(Communicated by David R. Larson)

\begin{abstract}
This paper deals with the connection between continuity of spectrum at an element $T$ of the Banach algebra of all bounded linear operators on a Banach space $X$ and at the adjoint $T^{*}$ of $T$. In particular, we show that, if $X$ is not reflexive, the spectrum function may be continuous at $T$ and discontinuous at $T^{*}$.
\end{abstract}

\section{INTRODUCTION}

We will denote by $\mathbb{K}_{\mathbb{C}}$ the set of all compact nonempty subsets of the complex plane $\mathbb{C}$, endowed with the Hausdorff metric $\Delta$. Recall that

$$
\Delta\left(K_{1}, K_{2}\right)=\max \left\{\max \left\{\operatorname{dist}\left(\lambda, K_{2}\right): \lambda \in K_{1}\right\}, \max \left\{\operatorname{dist}\left(\mu, K_{1}\right): \mu \in K_{2}\right\}\right\}
$$

for each $\left(K_{1}, K_{2}\right) \in \mathbb{K}_{\mathbb{C}} \times \mathbb{K}_{\mathbb{C}}$. If $\mathfrak{A}$ is a complex Banach algebra with identity, let $\sigma_{\mathfrak{A}}: \mathfrak{A} \rightarrow \mathbb{K}_{\mathbb{C}}$ and $r_{\mathfrak{A}}: \mathfrak{A} \rightarrow \mathbb{R}$ denote respectively the spectrum function on $\mathfrak{A}$ and the spectral radius function on $\mathfrak{A}$ (namely, the functions mapping respectively every $\mathfrak{a} \in \mathfrak{A}$ into its spectrum $\sigma_{\mathfrak{A}}(\mathfrak{a})$ and into its spectral radius $\left.r_{\mathfrak{A}}(\mathfrak{a})\right)$. Then the problem of continuity can be considered for each of $\sigma_{\mathfrak{A}}$ and $r_{\mathfrak{A}}$. Notice that

$$
\left|r_{\mathfrak{A}}(\mathfrak{a})-r_{\mathfrak{A}}(\mathfrak{b})\right| \leq \Delta\left(\sigma_{\mathfrak{A}}(\mathfrak{a}), \sigma_{\mathfrak{A}}(\mathfrak{b})\right) \quad \text { for every } \mathfrak{a}, \mathfrak{b} \in \mathfrak{A} .
$$

Hence, if $\sigma_{\mathfrak{A}}$ is continuous at $\mathfrak{a} \in \mathfrak{A}$, it follows that $r_{\mathfrak{A}}$ also is continuous at $\mathfrak{a}$.

It is well known that $\sigma_{\mathfrak{A}}$ is continuous on $\mathfrak{A}$ if $\mathfrak{A}$ is commutative or finitedimensional modulo the radical. An example - due to Kakutani-in the Banach algebra of all bounded linear operators on $\ell_{2}$ shows that even $r_{\mathfrak{A}}$ need not be continuous on the whole of $\mathfrak{A}$ if $\mathfrak{A}$ is neither commutative nor finite-dimensional modulo the radical (see [R], pp. 282-283).

If $X$ and $Y$ are Banach spaces over the same scalar field ( $\mathbb{R}$ or $\mathbb{C})$, let $L(X, Y)$ stand for the Banach space of all bounded linear operators from $X$ into $Y$. For each $T \in L(X, Y)$, we will denote by $T^{*}$ the adjoint of $T$. Thus, if we use the symbol $E^{*}$ to denote the dual space of any Banach space $E$, we have $T^{*} \in L\left(Y^{*}, X^{*}\right)$. For simplicity of notation, we will write $L(X)$ instead of $L(X, X)$. Also, we will denote by $I_{X}$ the identity operator on $X$. We remark that, if the Banach space $X$ is nonzero, then $L(X)$ is a Banach algebra with identity $I_{X}$. We will denote by $K(X)$ the ideal of compact operators on $X$.

Received by the editors March 12, 1998.

1991 Mathematics Subject Classification. Primary 47A10, 47C05.

Key words and phrases. Continuity of spectrum, adjoint operators in Banach spaces.

(C)1999 American Mathematical Society 
Notice that, if $X$ is a finite-dimensional nonzero complex Banach space, then the Banach algebras $L(X)$ and $L\left(X^{*}\right)$ have finite dimension. Hence $\sigma_{L(X)}$ and $\sigma_{L\left(X^{*}\right)}$ are continuous on $L(X)$ and $L\left(X^{*}\right)$, respectively.

The problem of characterizing the points of continuity of $\sigma_{\mathfrak{A}}$ and of $r_{\mathfrak{A}}$ is still open in the case of a general Banach algebra $\mathfrak{A}$. In the special cases of the Banach algebra $L(H)$ and of the quotient algebra $L(H) /_{K(H)}$ for an infinite-dimensional separable complex Hilbert space $H$, this problem was solved by J. B. Conway and B. B. Morrel (see [CM1], [CM2], [CM3]; notice that the Hilbert spaces in [CM3] are understood to be separable). Conway and Morrel's conditions for continuity of spectrum and spectral radius turn out to be sufficient for continuity of the corresponding functions also in the more general cases of the Banach algebras $L(X)$ and $L(X) /_{K(X)}$, where $X$ is an infinite-dimensional complex Banach space.

We also have been interested in continuity of spectrum and spectral radius. In particular, in [B1], 2.7, and in [B3], 1.1, we provided respectively two equivalent sufficient conditions for continuity of $\sigma_{\mathfrak{A}}$ and a sufficient condition for continuity of $r_{\mathfrak{A}}$ at a point $\mathfrak{a}$ of a complex Banach algebra $\mathfrak{A}$. In the special cases $\mathfrak{A}=L(H)$ and $\mathfrak{A}=L(H) /_{K(H)}$ (where $H$ is an infinite-dimensional separable complex Hilbert space), our conditions turn out to be equivalent to Conway and Morrel's, and thus necessary, as well as sufficient, for continuity of the corresponding functions. If $H$ is replaced by a general Banach space $X$, our conditions turn out to be less restrictive than Conway and Morrel's, both in $L(X)$ and in $L(X){ }_{K(X)}$. A general reference for these results is Section 3 of the survey article [B4].

Our main concern here is to investigate the relationship between continuity of $\sigma_{L(X)}$ at $T$ and continuity of $\sigma_{L\left(X^{*}\right)}$ at $T^{*}$ (where $X$ is a nonzero complex Banach space and $T \in L(X))$. The corresponding problem for the spectral radius function is also considered. Our interest in this problem was stimulated by an assertion we found in $[\mathrm{BE}]$ : indeed, in [BE], 3.4, the following is claimed to be a consequence of continuity of the function mapping each operator into its adjoint (actually, $[\mathrm{BE}]$ is in the context of unbounded operators; the assertion we quote here is derived from [BE], 3.4, by restricting the hypotheses to the case of a bounded operator).

Assertion 1.1. If $X$ is a nonzero complex Banach space and $\sigma_{L(X)}$ is continuous at $T \in L(X)$, then $\sigma_{L\left(X^{*}\right)}$ is continuous at $T^{*}$.

This seems clearly to be a misprint. Indeed, what follows easily from continuity of the adjoint function from $L(X)$ into $L\left(X^{*}\right)$ is actually the converse of Assertion 1.1 (see Proposition 3.1 below). However, one may ask whether Assertion 1.1 is nevertheless true. In this paper this question is answered in the negative.

In Section 2 we collect some preliminaries, in order to make our paper as selfcontained as possible.

Section 3 contains the results of this paper. We begin by observing that continuity of $\sigma_{L\left(X^{*}\right)}$ (respectively, $r_{L\left(X^{*}\right)}$ ) at $T^{*}$ implies continuity of $\sigma_{L(X)}$ (respectively, $\left.r_{L(X)}\right)$ at $T$, and the converse also holds if the Banach space $X$ is supposed to be reflexive (Proposition 3.1 and Corollary 3.2). Then we turn to our main result; namely, we prove that Assertion 1.1 is false: in Example 3.3 we construct a bounded linear operator $T$ on a nonreflexive Banach space, such that the spectrum function is continuous at $T$ and discontinuous at $T^{*}$. 
In order to prove continuity of spectrum at $T$, we appeal to a special case of [B1], 2.7, which is stated here in Section 2. We point out that Conway and Morrel's conditions for continuity of spectrum cannot be helpful in a situation like the one of Example 3.3. Indeed, they are "self-adjoint", in the sense that they are satisfied either by both an operator $A$ and its adjoint (in which case $\sigma_{L(X)}$ and $\sigma_{L\left(X^{*}\right)}$ are continuous at $A$ and $A^{*}$, respectively) or by none of them: this is apparent if you look at one of the several equivalent versions of these conditions, which requires the union of the set of all points of nonzero index of the semi-Fredholm domain and of the set of all complex numbers $\lambda$ such that $\{\lambda\}$ is a component of the spectrum to be dense in the spectrum (see Section 2 of [B4] for a discussion about Conway and Morrel's conditions in the Banach space setting).

At present, as we note in the concluding remarks of this paper, we do not know whether the analogue of Assertion 1.1 for the spectral radius is true or not.

\section{Preliminaries}

If $E, F$ are Banach spaces and $T \in L(E, F)$, we denote by $\mathcal{R}(T)$ and $\mathcal{N}(T)$ the range and the kernel of $T$, respectively.

Now let $X$ be a Banach space. We will call an element $P$ of $L(X)$ a projection of $X$ if $P^{2}=P$. It is well known that, if $P \in L(X)$ is a projection of $X$, then $\mathcal{R}(P)$ is a closed subspace of $X$ and moreover $X=\mathcal{R}(P) \oplus \mathcal{N}(P)$. Conversely, for each direct-sum decomposition $X=Y \oplus Z$, where $Y$ and $Z$ are closed subspaces of $X$, there exists a unique projection $P$ of $X$ satisfying $\mathcal{R}(P)=Y$ and $\mathcal{N}(P)=Z$. This projection will be called the projection of $X$ onto $Y$ along $Z$. Notice that $I_{X}-P$ is the projection of $X$ onto $Z$ along $Y$. Finally, we recall that a linear subspace $Y$ of $X$ is said to be complemented if it is the range of a projection of $X$ (or, equivalently, if $Y$ is closed and there exists a closed subspace $Z$ of $X$ such that $X=Y \oplus Z$ ).

The following characterization of the operators having complemented kernel and range will be useful in the sequel.

Theorem 2.1 (see [C], Chapter 1, Theorem 1). Let $X$ be a Banach space, and let $T \in L(X)$. Then the following two conditions are equivalent:

(i) both $\mathcal{N}(T)$ and $\mathcal{R}(T)$ are complemented subspaces of $X$;

(ii) there exists $S \in L(X)$ such that $T S T=T$ and $S T S=S$.

Moreover, if $T$ satisfies the two equivalent conditions above and $S \in L(X)$ is such that $T S T=T$ and $S T S=S$, then $\mathcal{N}(S)$ and $\mathcal{R}(S)$ are complemented subspaces of $X, X=\mathcal{R}(T) \oplus \mathcal{N}(S)=\mathcal{N}(T) \oplus \mathcal{R}(S)$, TS is the projection of $X$ onto $\mathcal{R}(T)$ along $\mathcal{N}(S)$ and $S T$ is the projection of $X$ onto $\mathcal{R}(S)$ along $\mathcal{N}(T)$.

We will also apply the following perturbation result.

Henceforth, the symbol $\sim$ will denote isomorphism between Banach spaces. We will write \|\|$_{X}$ for the norm of a Banach space $X$.

Theorem 2.2 (see [C], Chapter 5, Theorem 9 and Corollary). Let $X$ be a nonzero Banach space, and let $S, T \in L(X) \backslash\{0\}$ be such that $T S T=T$ and $S T S=S$ (which, by Theorem 2.1, forces $\mathcal{N}(T), \mathcal{R}(T), \mathcal{N}(S)$ and $\mathcal{R}(S)$ to be complemented subspaces of $X)$. If $\mathcal{N}(T) \subset \mathcal{R}\left(T^{n}\right)$ for every $n \in \mathbb{N}$, and $A \in L(X)$ is such that 
$A\left(\bigcap_{n \in \mathbb{N}} \mathcal{R}\left(T^{n}\right)\right) \subset \bigcap_{n \in \mathbb{N}} \mathcal{R}\left(T^{n}\right)$ and $\|A\|_{L(X)}<\frac{1}{\|S\|_{L(X)}}$, then $T-A$ has complemented kernel and range and satisfies $X=\mathcal{R}(T-A) \oplus \mathcal{N}(S)=\mathcal{N}(T-A) \oplus \mathcal{R}(S)$

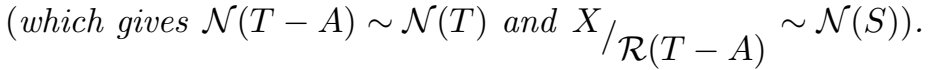

From now on, the closure and the boundary of a subset $\Sigma$ of a topological space will be denoted by $\bar{\Sigma}$ and $\partial \Sigma$, respectively.

For each Banach algebra $\mathfrak{A}$ with identity, let $\mathcal{G}_{\mathfrak{A}}$ denote the group of invertible elements of $\mathfrak{A}$. The following characterization of membership in $\overline{\mathcal{G}}_{L(X)}$ for a bounded linear operator - having complemented kernel and range - on a Banach space $X$ was obtained by M. Gonzalez in [G].

Theorem 2.3 (see $[\mathrm{G}], \S 1$ ). Let $X$ be a Banach space, and let $T \in L(X)$ have complemented kernel and range. Then $T \in \overline{\mathcal{G}}_{L(X)}$ if and only if $\mathcal{N}(T) \sim X_{/ \mathcal{R}(T)}$.

Let $\mathfrak{A}$ be a complex Banach algebra with identity $\mathfrak{e}$. For each $\mathfrak{a} \in \mathfrak{A}$, we set

$$
\mathcal{S}(\mathfrak{a})=\left\{\lambda \in \mathbb{C}: \lambda \mathfrak{e}-\mathfrak{a} \notin \overline{\mathcal{G}}_{\mathfrak{A}}\right\} .
$$

Since $\mathfrak{A} \backslash \overline{\mathcal{G}}_{\mathfrak{A}}$ is an open subset of $\mathfrak{A}$, it follows that $\mathcal{S}(\mathfrak{a})$ is an open subset of $\mathbb{C}$. Furthermore, we have $\overline{\mathcal{S}(\mathfrak{a})} \subset \sigma_{\mathfrak{A}}(\mathfrak{a})$.

For our purposes here, it is sufficient to state the following straightforward consequence of [B1], 2.7.

Theorem 2.4. Let $\mathfrak{A}$ be a complex Banach algebra with identity. If $\mathfrak{a} \in \mathfrak{A}$ satisfies $\sigma_{\mathfrak{A}}(\mathfrak{a})=\overline{\mathcal{S}(\mathfrak{a})}$, then $\sigma_{\mathfrak{A}}$ is continuous at $\mathfrak{a}$.

Finally, we will more than once make use of the following well known result, relating the kernels and ranges of an operator and of its adjoint.

Henceforth, the annihilator of a subspace $M$ of a Banach space $X$ in $X^{*}$ (see [TL], Definition on page 163) will be denoted by $M^{\circ}$.

Theorem 2.5 (see [TL], IV, 8.4 and 10.1). Let $X, Y$ be Banach spaces, and let $T \in L(X, Y)$. Then:

(i) $\mathcal{N}\left(T^{*}\right)=(\mathcal{R}(T))^{\circ}$;

(ii) $\mathcal{R}(T)$ is closed if and only if $\mathcal{R}\left(T^{*}\right)$ is closed;

(iii) if $\mathcal{R}(T)$ is closed, we have $\mathcal{R}\left(T^{*}\right)=(\mathcal{N}(T))^{\circ}$.

\section{The exAmple}

We begin by establishing the relationship between continuity of spectrum (respectively, spectral radius) at a bounded linear operator $T$ on a Banach space and continuity of spectrum (respectively, spectral radius) at $T^{*}$.

Proposition 3.1. Let $X$ be a nonzero complex Banach space, and let $T \in L(X)$.

(i) If $\sigma_{L\left(X^{*}\right)}$ is continuous at $T^{*}$, then $\sigma_{L(X)}$ is continuous at $T$.

(ii) If $r_{L\left(X^{*}\right)}$ is continuous at $T^{*}$, then $r_{L(X)}$ is continuous at $T$.

Proof. It suffices to remark that $\sigma_{L(X)}=\sigma_{L\left(X^{*}\right)} \circ \Phi_{X}$ and $r_{L(X)}=r_{L\left(X^{*}\right)} \circ \Phi_{X}$, where

$$
\Phi_{X}: L(X) \ni A \mapsto A^{*} \in L\left(X^{*}\right) .
$$

Since the linear map $\Phi_{X}$ is an isometry, and consequently is continuous, we get the desired result. 
If the Banach space $X$ is assumed to be reflexive, then the converse of each of assertions (i) and (ii) of Proposition 3.1 also holds, as the following result shows.

Corollary 3.2. Let $X$ be a nonzero reflexive complex Banach space, and let $T \in$ $L(X)$.

(i) If $\sigma_{L(X)}$ is continuous at $T$, then $\sigma_{L\left(X^{*}\right)}$ is continuous at $T^{*}$.

(ii) If $r_{L(X)}$ is continuous at $T$, then $r_{L\left(X^{*}\right)}$ is continuous at $T^{*}$.

Proof. Since $X$ is reflexive, the map

$$
\Psi_{X}: L(X) \ni A \mapsto A^{* *} \in L\left(X^{* *}\right)
$$

is an isometric isomorphism of Banach algebras. Hence continuity of $\sigma_{L(X)}$ (respectively, $\left.r_{L(X)}\right)$ at $T$ implies continuity of $\sigma_{L\left(X^{* *}\right)}\left(\right.$ respectively, $\left.r_{L\left(X^{* *}\right)}\right)$ at $T^{* *}$, which gives continuity of $\sigma_{L\left(X^{*}\right)}$ (respectively, $\left.r_{L\left(X^{*}\right)}\right)$ at $T^{*}$ by Proposition 3.1.

The following example shows that, at least as concerns assertion (i), reflexivity of $X$ cannot be removed from the hypotheses of Corollary 3.2.

Example 3.3. We begin by considering the bounded linear operators $V$ and $W$ on the complex Banach space $\ell_{\infty}$, defined by

$$
V\left(y_{n}\right)_{n \in \mathbb{N}}=\left(y_{2 n}\right)_{n \in \mathbb{N}} \quad \text { for each }\left(y_{n}\right)_{n \in \mathbb{N}} \in \ell_{\infty}
$$

and

$$
W\left(z_{n}\right)_{n \in \mathbb{N}}=\left(\zeta_{n}\right)_{n \in \mathbb{N}}\left(\text { where } \zeta_{k}=\left\{\begin{array}{ll}
z_{\frac{k}{2}} & \text { if } k \text { is even } \\
0 & \text { if } k \text { is odd }
\end{array} \quad \text { for each } k \in \mathbb{N}\right)\right.
$$

for each $\left(z_{n}\right)_{n \in \mathbb{N}} \in \ell_{\infty}$. Notice that each of $V$ and $W$ leaves $c_{0}$ invariant. Then two bounded linear operators $S$ and $U$ on $c_{0}$ can be defined as follows:

$$
S\left(x_{n}\right)_{n \in \mathbb{N}}=V\left(x_{n}\right)_{n \in \mathbb{N}} \quad \text { and } \quad U\left(x_{n}\right)_{n \in \mathbb{N}}=W\left(x_{n}\right)_{n \in \mathbb{N}} \quad \text { for each }\left(x_{n}\right)_{n \in \mathbb{N}} \in c_{0} .
$$

Now let $E$ denote the complex Banach space $c_{0} \times \ell_{\infty} \times \ell_{\infty}$, endowed with the norm \|\|$_{E}$ defined by

$$
\|(x, y, z)\|_{E}=\max \left\{\|x\|_{c_{0}},\|y\|_{\ell_{\infty}},\|z\|_{\ell_{\infty}}\right\} \quad \text { for each }(x, y, z) \in c_{0} \times \ell_{\infty} \times \ell_{\infty} .
$$

We define a bounded linear operator $T$ on $E$ as follows:

$$
T(x, y, z)=(S x, V y, W z) \quad \text { for each }(x, y, z) \in E .
$$

We prove that the spectrum function is continuous at $T$ and discontinuous at $T^{*}$.

We first prove that $\sigma_{L(E)}$ is continuous at $T$.

We begin by remarking that $S U=I_{c_{0}}$ and $V W=I_{\ell_{\infty}}$. Then $\mathcal{R}(S)=c_{0}$, $\mathcal{N}(U)=\{0\}, \mathcal{R}(V)=\ell_{\infty}$ and $\mathcal{N}(W)=\{0\}$. Furthermore, if $R \in L(E)$ is defined by

$$
R(x, y, z)=(U x, W y, V z) \quad \text { for each }(x, y, z) \in E,
$$

we see that for every $(x, y, z) \in E$ we have

$$
T R T(x, y, z)=(S U S x, V W V y, W V W z)=(S x, V y, W z)=T(x, y, z)
$$

and

$$
R T R(x, y, z)=(U S U x, W V W y, V W V z)=(U x, W y, V z)=R(x, y, z) .
$$

Hence $T R T=T$ and $R T R=R$. Consequently, by Theorem 2.1, each of $\mathcal{N}(T)$, $\mathcal{R}(T), \mathcal{N}(R)$ and $\mathcal{R}(R)$ is a complemented subspace of $E$. From now on, to shorten notation, we will let $F$ and $G$ stand for $\mathcal{R}(R)$ and $\mathcal{N}(R)$, respectively. 
It is easily seen that

$$
T^{n}(x, y, z)=\left(S^{n} x, V^{n} y, W^{n} z\right) \quad \text { for each }(x, y, z) \in E \text { and for each } n \in \mathbb{N} .
$$

Hence for every $n \in \mathbb{N}$ we obtain

$$
\begin{aligned}
\mathcal{R}\left(T^{n}\right) & =\mathcal{R}\left(S^{n}\right) \times \mathcal{R}\left(V^{n}\right) \times \mathcal{R}\left(W^{n}\right) \\
& =c_{0} \times \ell_{\infty} \times \mathcal{R}\left(W^{n}\right) \supset \mathcal{N}(S) \times \mathcal{N}(V) \times\{0\}=\mathcal{N}(S) \times \mathcal{N}(V) \times \mathcal{N}(W)=\mathcal{N}(T) .
\end{aligned}
$$

We remark that $\|S\|_{L\left(c_{0}\right)}=\|U\|_{L\left(c_{0}\right)}=\|V\|_{L\left(\ell_{\infty}\right)}=\|W\|_{L\left(\ell_{\infty}\right)}=1$. Then it is not difficult to check that $\|T\|_{L(E)}=\|R\|_{L(E)}=1$.

Since $\|R\|_{L(E)}=1$, applying Theorem 2.2 with $A=\lambda I_{E}$ we conclude that, for each $\lambda$ in the open unit ball $\mathbf{B}$ of the complex plane, both $\mathcal{N}\left(\lambda I_{E}-T\right)$ and $\mathcal{R}\left(\lambda I_{E}-T\right)$ are complemented subspaces of $E$, and moreover we have

$$
\mathcal{N}\left(\lambda I_{E}-T\right) \sim \mathcal{N}(T)=\mathcal{N}(S) \times \mathcal{N}(V) \times\{0\} \sim \mathcal{N}(S) \times \mathcal{N}(V)
$$

and

$$
E_{/ \mathcal{R}\left(\lambda I_{E}-T\right)} \sim G=\mathcal{N}(U) \times \mathcal{N}(W) \times \mathcal{N}(V)=\{0\} \times\{0\} \times \mathcal{N}(V) \sim \mathcal{N}(V)
$$

Since

$$
\mathcal{N}(S)=\left\{\left(x_{n}\right)_{n \in \mathbb{N}} \in c_{0}: x_{2 k}=0 \text { for each } k \in \mathbb{N}\right\} \sim c_{0}
$$

and

$$
\mathcal{N}(V)=\left\{\left(y_{n}\right)_{n \in \mathbb{N}} \in \ell_{\infty}: y_{2 k}=0 \text { for each } k \in \mathbb{N}\right\} \sim \ell_{\infty},
$$

it follows that

$$
\mathcal{N}\left(\lambda I_{E}-T\right) \sim c_{0} \times \ell_{\infty} \quad \text { and } \quad E_{/_{\mathcal{R}}\left(\lambda I_{E}-T\right)} \sim \ell_{\infty} \quad \text { for each } \lambda \in \mathbf{B} .
$$

Now let $\lambda \in \mathbf{B}$. Since $c_{0}$ is isomorphic to no complemented subspace of $\ell_{\infty}$ (see [LT], 2.a.7), it follows that $\mathcal{N}\left(\lambda I_{E}-T\right)$ is not isomorphic to $E_{/ \mathcal{R}}\left(\lambda I_{E}-T\right)$. Now we can apply Theorem 2.3 and concude that $\lambda I_{E}-T \notin \overline{\mathcal{G}}_{L(E)}$.

We have thus proved that $\mathbf{B} \subset \mathcal{S}(T)$. On the other hand, since $\|T\|_{L(E)}=1$, it follows that $\sigma_{L(E)}(T) \subset \overline{\mathbf{B}}$. Since $\mathcal{S}(T)$ is an open subset of $\mathbb{C}$, contained in $\sigma_{L(E)}(T)$, we obtain

$$
\mathcal{S}(T)=\mathbf{B} \quad \text { and } \quad \sigma_{L(E)}(T)=\overline{\mathbf{B}} .
$$

Hence $\sigma_{L(E)}(T)=\overline{\mathcal{S}(T)}$, and consequently, by Theorem $2.4, \sigma_{L(E)}$ is continuous at $T$.

We point out that $T^{*}$ does not satisfy the sufficient condition for continuity of spectrum given in Theorem 2.4. Indeed, since $\sigma_{L\left(E^{*}\right)}\left(T^{*}\right)=\sigma_{L(E)}(T)=\overline{\mathbf{B}}$, it follows that $\mathcal{S}\left(T^{*}\right) \subset \mathbf{B}$. Furthermore, for each $\lambda \in \mathbf{B}$, since $\mathcal{N}\left(\lambda I_{E}-T\right)$ and $\mathcal{R}\left(\lambda I_{E}-T\right)$ are complemented subspaces of $E$, by applying Theorem 2.1 (see also $[\mathrm{C}], \S 1.2$, Remark (3)) we can assert that $\mathcal{N}\left(\lambda I_{E^{*}}-T^{*}\right)$ and $\mathcal{R}\left(\lambda I_{E^{*}}-T^{*}\right)$ are complemented subspaces of $E^{*}$. Finally, by Theorem 2.5 and [TL], III, 3.3, we have

$$
\mathcal{N}\left(\lambda I_{E^{*}}-T^{*}\right)=\left(\mathcal{R}\left(\lambda I_{E}-T\right)\right)^{\circ} \sim\left(E_{/ \mathcal{R}\left(\lambda I_{E}-T\right)}\right)^{*} \sim \ell_{\infty}^{*}
$$


and

$$
E^{*} /_{\mathcal{R}\left(\lambda I_{E^{*}}-T^{*}\right)}=E^{*} /\left(\mathcal{N}\left(\lambda I_{E}-T\right)\right)^{\circ} \sim\left(\mathcal{N}\left(\lambda I_{E}-T\right)\right)^{*} \sim\left(c_{0} \times \ell_{\infty}\right)^{*} \sim \ell_{1} \times \ell_{\infty}^{*} .
$$

Since every dual space is complemented in its bidual (see $[\mathrm{H}], 5.9 .4)$, it follows that $\ell_{1}$ is isomorphic to a complemented subspace of $\ell_{\infty}^{*}$. From this, since $\ell_{1}$ is easily seen to be isomorphic to its square, we can derive that $\ell_{1} \times \ell_{\infty}^{*}$ is isomorphic to $\ell_{\infty}^{*}$, and consequently

$$
\mathcal{N}\left(\lambda I_{E^{*}}-T^{*}\right) \sim E^{*} / \mathcal{R}\left(\lambda I_{E^{*}}-T^{*}\right) \quad \text { for each } \lambda \in \mathbf{B} .
$$

Now Theorem 2.3 yields $\mathcal{S}\left(T^{*}\right) \cap \mathbf{B}=\emptyset$, which, since $\mathcal{S}\left(T^{*}\right) \subset \mathbf{B}$, gives $\mathcal{S}\left(T^{*}\right)=\emptyset$. Hence $T^{*}$ does not satisfy the condition of Theorem 2.4 .

Now we prove that the spectrum function is actually discontinuous at $T^{*}$.

From Theorem 2.2 it follows also that

$$
E=\mathcal{N}\left(\lambda I_{E}-T\right) \oplus F=\mathcal{R}\left(\lambda I_{E}-T\right) \oplus G \quad \text { for each } \lambda \in \mathbf{B} .
$$

For each $\lambda \in \mathbf{B}$, let $P_{\lambda}$ and $Q_{\lambda}$ denote the projection of $E$ onto $\mathcal{N}\left(\lambda I_{E}-T\right)$ along $F$ and the projection of $E$ onto $G$ along $\mathcal{R}\left(\lambda I_{E}-T\right)$, respectively. From Theorem 2.5 it follows that, for each $\lambda \in \mathbf{B}, P_{\lambda}^{*}$ is the projection of $E^{*}$ onto $F^{\circ}$ along $\mathcal{R}\left(\lambda I_{E^{*}}-T^{*}\right)$ and $Q_{\lambda}^{*}$ is the projection of $E^{*}$ onto $\mathcal{N}\left(\lambda I_{E^{*}}-T^{*}\right)$ along $G^{\circ}$. Consequently, we have

$$
E^{*}=\mathcal{N}\left(\lambda I_{E^{*}}-T^{*}\right) \oplus G^{\circ}=\mathcal{R}\left(\lambda I_{E^{*}}-T^{*}\right) \oplus F^{\circ} \quad \text { for each } \lambda \in \mathbf{B} .
$$

In particular, we deduce that

$$
E^{*}=\mathcal{N}\left(T^{*}\right) \oplus G^{\circ}=\mathcal{R}\left(T^{*}\right) \oplus F^{\circ},
$$

$P_{0}^{*}$ is the projection of $E^{*}$ onto $F^{\circ}$ along $\mathcal{R}\left(T^{*}\right)$ and $Q_{0}^{*}$ is the projection of $E^{*}$ onto $\mathcal{N}\left(T^{*}\right)$ along $G^{\circ}$.

Since $\mathcal{N}\left(T^{*}\right)$ is isomorphic to $E^{*} / \mathcal{R}\left(T^{*}\right)$, and consequently to $F^{\circ}$, there exists $\Gamma \in L\left(\mathcal{N}\left(T^{*}\right), F^{\circ}\right)$ such that $\mathcal{N}(\Gamma)=\{0\}$ and $\mathcal{R}(\Gamma)=F^{\circ}$. Furthermore, without loss of generality we can assume that $\|\Gamma\|_{L\left(\mathcal{N}\left(T^{*}\right), F^{\circ}\right)}=1$. For each $n \in \mathbb{N}$, let $A_{n} \in L\left(E^{*}\right)$ be defined by

$$
A_{n} \xi^{*}=T^{*} \xi^{*}-\frac{1}{n+1} \Gamma Q_{0}^{*} \xi^{*} \quad \text { for each } \xi^{*} \in E^{*} .
$$

Clearly, $A_{n}$ converges to $T^{*}$ in $L\left(E^{*}\right)$ as $n \rightarrow+\infty$.

We prove that $\sigma_{L\left(E^{*}\right)}\left(A_{n}\right)=\partial \mathbf{B}$ for each $n \in \mathbb{N}$.

From Theorem 2.1 it follows that $R T=I_{E}-P_{0}$ and $T R=I_{E}-Q_{0}$. Since $S U=I_{c_{0}}$ and $V W=I_{\ell_{\infty}}$, for each $(x, y, z) \in E$ we obtain

$$
\left(I_{E}-P_{0}\right)(x, y, z)=(U S x, W V y, z) \quad \text { and } \quad\left(I_{E}-Q_{0}\right)(x, y, z)=(x, y, W V z) \text {. }
$$

We remark that, if we set $\delta_{n}=\left\{\begin{array}{ll}1 & \text { if } n \text { is even } \\ 0 & \text { if } n \text { is odd }\end{array}\right.$ for each $n \in \mathbb{N}$, we have

$$
U S\left(x_{n}\right)_{n \in \mathbb{N}}=\left(\delta_{n} x_{n}\right)_{n \in \mathbb{N}} \quad \text { for each }\left(x_{n}\right)_{n \in \mathbb{N}} \in c_{0}
$$

and

$$
W V\left(y_{n}\right)_{n \in \mathbb{N}}=\left(\delta_{n} y_{n}\right)_{n \in \mathbb{N}} \text { for each }\left(y_{n}\right)_{n \in \mathbb{N}} \in \ell_{\infty}
$$


Now let $\xi, \zeta \in E$. If $\left(x_{n}\right)_{n \in \mathbb{N}},\left(u_{n}\right)_{n \in \mathbb{N}} \in c_{0},\left(y_{n}\right)_{n \in \mathbb{N}},\left(z_{n}\right)_{n \in \mathbb{N}},\left(v_{n}\right)_{n \in \mathbb{N}},\left(w_{n}\right)_{n \in \mathbb{N}} \in$ $\ell_{\infty}$ satisfy $\xi=\left(\left(x_{n}\right)_{n \in \mathbb{N}},\left(y_{n}\right)_{n \in \mathbb{N}},\left(z_{n}\right)_{n \in \mathbb{N}}\right)$ and $\zeta=\left(\left(u_{n}\right)_{n \in \mathbb{N}},\left(v_{n}\right)_{n \in \mathbb{N}},\left(w_{n}\right)_{n \in \mathbb{N}}\right)$, we obtain

$$
\begin{aligned}
& \left\|P_{0} \xi+\left(I_{E}-P_{0}\right) \zeta\right\|_{E} \\
& \quad=\left\|\left(\left(\left(1-\delta_{n}\right) x_{n}+\delta_{n} u_{n}\right)_{n \in \mathbb{N}},\left(\left(1-\delta_{n}\right) y_{n}+\delta_{n} v_{n}\right)_{n \in \mathbb{N}},\left(w_{n}\right)_{n \in \mathbb{N}}\right)\right\|_{E} \\
& \quad=\max \left\{\left\|\left(\left(1-\delta_{n}\right) x_{n}+\delta_{n} u_{n}\right)_{n \in \mathbb{N}}\right\|_{c_{0}},\left\|\left(\left(1-\delta_{n}\right) y_{n}+\delta_{n} v_{n}\right)_{n \in \mathbb{N}}\right\|_{\ell_{\infty}},\left\|\left(w_{n}\right)_{n \in \mathbb{N}}\right\|_{\ell_{\infty}}\right\} \\
& \quad \leq \max \left\{\|\xi\|_{E},\|\zeta\|_{E}\right\}
\end{aligned}
$$

and

$$
\begin{aligned}
\| Q_{0} \xi & +\left(I_{E}-Q_{0}\right) \zeta \|_{E} \\
& =\left\|\left(\left(u_{n}\right)_{n \in \mathbb{N}},\left(v_{n}\right)_{n \in \mathbb{N}},\left(\left(1-\delta_{n}\right) z_{n}+\delta_{n} w_{n}\right)_{n \in \mathbb{N}}\right)\right\|_{E} \\
& =\max \left\{\left\|\left(u_{n}\right)_{n \in \mathbb{N}}\right\|_{c_{0}},\left\|\left(v_{n}\right)_{n \in \mathbb{N}}\right\|_{\ell_{\infty}},\left\|\left(\left(1-\delta_{n}\right) z_{n}+\delta_{n} w_{n}\right)_{n \in \mathbb{N}}\right\|_{\ell_{\infty}}\right\} \\
& \leq \max \left\{\|\xi\|_{E},\|\zeta\|_{E}\right\} .
\end{aligned}
$$

From this, applying [HWW], I, comments following 1.1, we conclude that

$$
\left\|\xi^{*}\right\|_{E^{*}}=\left\|P_{0}^{*} \xi^{*}\right\|_{E^{*}}+\left\|\xi^{*}-P_{0}^{*} \xi^{*}\right\|_{E^{*}}=\left\|Q_{0}^{*} \xi^{*}\right\|_{E^{*}}+\left\|\xi^{*}-Q_{0}^{*} \xi^{*}\right\|_{E^{*}}
$$

for each $\xi^{*} \in E^{*}$. Consequently, for each $\xi^{*} \in E^{*}$ we have

$$
\begin{aligned}
& \left\|A_{n} \xi^{*}\right\|_{E^{*}}=\left\|P_{0}^{*} A_{n} \xi^{*}\right\|_{E^{*}}+\left\|A_{n} \xi^{*}-P_{0}^{*} A_{n} \xi^{*}\right\|_{E^{*}}=\frac{\left\|\Gamma Q_{0}^{*} \xi^{*}\right\|_{E^{*}}}{n+1}+\left\|T^{*} \xi^{*}\right\|_{E^{*}} \\
& =\frac{\left\|\Gamma Q_{0}^{*} \xi^{*}\right\|_{E^{*}}}{n+1}+\left\|T^{*}\left(\xi^{*}-Q_{0}^{*} \xi^{*}\right)\right\|_{E^{*}} \leq\left\|Q_{0}^{*} \xi^{*}\right\|_{E^{*}}+\left\|\xi^{*}-Q_{0}^{*} \xi^{*}\right\|_{E^{*}}=\left\|\xi^{*}\right\|_{E^{*}} .
\end{aligned}
$$

Hence $\left\|A_{n}\right\|_{L\left(E^{*}\right)} \leq 1$ for every $n \in \mathbb{N}$, which gives $\sigma_{L\left(E^{*}\right)}\left(A_{n}\right) \subset \overline{\mathbf{B}}$ for every $n \in \mathbb{N}$.

Now let $n \in \mathbb{N}, \lambda \in \mathbf{B}$. We remark that

$$
\left(\lambda I_{E^{*}}-A_{n}\right) \xi^{*}=\left(\lambda I_{E^{*}}-T^{*}\right) \xi^{*}+\frac{1}{n+1} \Gamma Q_{0}^{*} \xi^{*} \quad \text { for each } \xi^{*} \in E^{*} .
$$

Since $\mathcal{R}\left(\lambda I_{E^{*}}-T^{*}\right) \cap \mathcal{R}(\Gamma)=\mathcal{R}\left(\lambda I_{E^{*}}-T^{*}\right) \cap F^{\circ}=\{0\}$, it follows that

$$
\mathcal{N}\left(\lambda I_{E^{*}}-A_{n}\right)=\mathcal{N}\left(\lambda I_{E^{*}}-T^{*}\right) \cap \mathcal{N}\left(\Gamma Q_{0}^{*}\right)=\mathcal{N}\left(\lambda I_{E^{*}}-T^{*}\right) \cap G^{\circ}=\{0\} .
$$

Since $G^{\circ}=\mathcal{N}\left(Q_{0}^{*}\right)$ and $E^{*}=\mathcal{N}\left(\lambda I_{E^{*}}-T^{*}\right) \oplus G^{\circ}$, we obtain

$$
\mathcal{R}\left(\lambda I_{E^{*}}-A_{n}\right) \supset\left(\lambda I_{E^{*}}-A_{n}\right)\left(G^{\circ}\right)=\left(\lambda I_{E^{*}}-T^{*}\right)\left(G^{\circ}\right)=\mathcal{R}\left(\lambda I_{E^{*}}-T^{*}\right) .
$$

Hence

$$
F^{\circ}=\mathcal{R}\left(\Gamma Q_{0}^{*}\right) \subset \mathcal{R}\left(\lambda I_{E^{*}}-A_{n}\right)+\mathcal{R}\left(\lambda I_{E^{*}}-T^{*}\right)=\mathcal{R}\left(\lambda I_{E^{*}}-A_{n}\right) .
$$

This, since $E^{*}=\mathcal{R}\left(\lambda I_{E^{*}}-T^{*}\right) \oplus F^{\circ}$, gives $\mathcal{R}\left(\lambda I_{E^{*}}-A_{n}\right)=E^{*}$. Therefore, $\lambda \notin \sigma_{L\left(E^{*}\right)}\left(A_{n}\right)$.

We have thus proved that $\sigma_{L\left(E^{*}\right)}\left(A_{n}\right) \subset \partial \mathbf{B}$ for every $n \in \mathbb{N}$ (notice that this, together with $\left\|A_{n}\right\|_{L\left(E^{*}\right)} \leq 1$ for every $n \in \mathbb{N}$, yields $\left\|A_{n}\right\|_{L\left(E^{*}\right)}=1$ for every $n \in \mathbb{N})$.

It remains to prove the opposite inclusion. To this end, we begin by showing that $\sigma_{L\left(\ell_{\infty}\right)}(W)=\overline{\mathbf{B}}$. We first remark that $\ell_{\infty}=\mathcal{R}\left(\mu I_{\ell_{\infty}}-W\right) \oplus \mathcal{N}(V)$ for every 
$\mu \in \mathbf{B}$ (this can be obtained either from the decomposition $E=\mathcal{R}\left(\mu I_{E}-T\right) \oplus G$, or by applying Theorem 2.2 with $\left.A=\mu I_{\ell_{\infty}}\right)$. Since $\mathcal{N}(V) \neq\{0\}$, it follows that $\sigma_{L\left(\ell_{\infty}\right)}(W) \supset \mathbf{B}$. On the other hand, $\|W\|_{L\left(\ell_{\infty}\right)}=1$ gives $\sigma_{L\left(\ell_{\infty}\right)}(W) \subset \overline{\mathbf{B}}$, from which we derive the desired equality.

Now let $\lambda \in \partial \mathbf{B}$. Since $\lambda \in \partial \sigma_{L\left(\ell_{\infty}\right)}(W)$, it follows that $\lambda I_{\ell_{\infty}}-W$ is not an injective operator with closed range (see [TL], V, 4.1). Hence, if we consider the bounded linear operator

$$
J: \ell_{\infty} \ni z \mapsto(0,0, z) \in E,
$$

we can assert that $J\left(\lambda I_{\ell_{\infty}}-W\right)$ is not an injective operator with closed range, either. It is easily seen that $\left(\lambda I_{E}-T\right) J=J\left(\lambda I_{\ell_{\infty}}-W\right)$. Since $\left(\lambda I_{E}-T\right) J$ is not an injective operator with closed range, from Theorem 2.5 it follows that $J^{*}\left(\lambda I_{E^{*}}-T^{*}\right)$ is not surjective. On the other hand, since

$$
\mathcal{R}(J)=\{0\} \times\{0\} \times \ell_{\infty} \subset \mathcal{R}(U) \times \mathcal{R}(W) \times \ell_{\infty}=\mathcal{R}(U) \times \mathcal{R}(W) \times \mathcal{R}(V)=F,
$$

applying Theorem 2.5 we obtain

$$
\mathcal{N}\left(J^{*}\right)=(\mathcal{R}(J))^{\circ} \supset F^{\circ}=\mathcal{R}(\Gamma) .
$$

Hence

$$
J^{*}\left(\lambda I_{E^{*}}-A_{n}\right)=J^{*}\left(\lambda I_{E^{*}}-T^{*}\right)
$$

for every $n \in \mathbb{N}$. We conclude that $J^{*}\left(\lambda I_{E^{*}}-A_{n}\right)$ is surjective for no $n \in \mathbb{N}$. Since $J$ is injective and has closed range, and consequently, by Theorem $2.5, J^{*}$ is surjective, it follows that $\lambda I_{E^{*}}-A_{n}$ is surjective for no $n \in \mathbb{N}$. Hence $\lambda \in \sigma_{L\left(E^{*}\right)}\left(A_{n}\right)$ for every $n \in \mathbb{N}$. The desired equality $\sigma_{L\left(E^{*}\right)}\left(A_{n}\right)=\partial \mathbf{B}$ is now established for every $n \in \mathbb{N}$.

Now, in order to prove that $\sigma_{L\left(E^{*}\right)}$ is discontinuous at $T^{*}$, it suffices to observe that

$$
\Delta\left(\sigma_{L\left(E^{*}\right)}\left(A_{n}\right), \sigma_{L\left(E^{*}\right)}\left(T^{*}\right)\right)=\Delta(\partial \mathbf{B}, \overline{\mathbf{B}})=1 \quad \text { for each } n \in \mathbb{N},
$$

although $A_{n} \rightarrow T^{*}$ as $n \rightarrow+\infty$.

In [B2] we had already used the fact that $c_{0} \times \ell_{\infty}$ and $\ell_{\infty}$ are nonisomorphic Banach spaces having isomorphic duals, in order to construct an operator $T \in$ $L\left(c_{0} \times \ell_{\infty}\right)$, having complemented kernel and range, such that $T \notin \overline{\mathcal{G}}_{L\left(c_{0} \times \ell_{\infty}\right)}$ and $T^{*} \in \overline{\mathcal{G}}_{L\left(\left(c_{0} \times \ell_{\infty}\right)^{*}\right)}$ (see [B2], 1.6). We point out that the operator $T$ of [B2], 1.6 would not have been suitable for our purposes in Example 3.3 here. Indeed, it can be proved that $\sigma_{L\left(c_{0} \times \ell_{\infty}\right)}(T)=\overline{\mathbf{B}}$ and $\mathcal{S}\left(T^{*}\right)=\mathbf{B} \backslash\{0\}$. Consequently, by Theorem 2.4 and Proposition 3.1, the spectrum function is continuous at $T^{*}$ as well as it is so at $T$.

We conclude by remarking that the sequence $\left(A_{n}\right)_{n \in \mathbb{N}}$, constructed in Example 3.3 , does not yield an example of discontinuity of $r_{L\left(E^{*}\right)}$ at the adjoint of an operator at which $r_{L(E)}$ is continuous, not even if we add a multiple of the identity to its terms (notice that adding a multiple of the identity to $T$ preserves continuity of $\sigma_{L(E)}$, which in turn gives continuity of $\left.r_{L(E)}\right)$. Indeed, for each $\nu \in \mathbb{C}$, since $\sigma\left(A_{n}\right)=\partial \mathbf{B}$ for every $n \in \mathbb{N}$, it follows that

$$
r_{L\left(E^{*}\right)}\left(A_{n}+\nu I_{E^{*}}\right)=|\nu|+1=r_{L\left(E^{*}\right)}\left(T^{*}+\nu I_{E^{*}}\right) \quad \text { for every } n \in \mathbb{N} .
$$

Therefore Example 3.3 leaves open the problem whether continuity of spectral radius at an operator $A$ implies continuity of spectral radius at $A^{*}$. 


\section{REFERENCES}

[B1] L.BURLANDO, Spectral continuity, Atti Sem. Mat. Fis. Univ. Modena 40 (1992), 591605. MR 94a: 46063

[B2] L. BURLANDO, Generalized semi-Fredholm operators that belong to the closure of the group of invertible operators, Math. Balkanica (N. S.) 6 (1992), 223-230. MR 93h:47011

[B3] L. BURLANDO, Spectral continuity in some Banach algebras, Rocky Mountain J. Math. 23 (1993), 17-39. MR 94c:46092

[B4] L. BURLANDO, Continuity of spectrum and spectral radius in Banach algebras, Functional Analysis and Operator Theory (J. Zemánek, ed.), Banach Center Publ., vol. 30, Institute of Mathematics, Polish Academy of Sciences, Warsaw, 1994, pp. 53-100. MR 95i:46062

[BE] N. J. BEZAK and M. EISEN, Continuity properties of operator spectra, Canad. J. Math. 29 (1977), 429-437. MR 55:1100

[C] S. R. CARADUS, Generalized Inverses and Operator Theory, Queen's Papers in Pure and Appl. Math., vol. 50, Queen's University, Kingston, Ontario, 1978. MR 81m:47003

[CM1] J. B. CONWAY and B. B. MORREL, Operators that are points of spectral continuity, Integral Equations Operator Theory 2 (1979), 174-198. MR 80h:47004

[CM2] J. B. CONWAY and B. B. MORREL, Operators that are points of spectral continuity, II, Integral Equations Operator Theory 4 (1981), 459-503. MR 83i:47007

[CM3] J. B. CONWAY and B. B. MORREL, Behaviour of the spectrum under small perturbations, Proc. Roy. Irish Acad. Sect. A 81 (1981), 55-63. MR 83a:47002

[G] M. GONZALEZ, A perturbation result for generalised Fredholm operators in the boundary of the group of invertible operators, Proc. Roy. Irish Acad. Sect. A 86 (1986), 123-126. MR 88g:47023

[H] R. HARTE, Invertibility and Singularity for Bounded Linear Operators, Marcel Dekker, Inc., New York-Basel, 1988. MR 89d:47001

[HWW] P. HARMAND, D. WERNER and W. WERNER, $M$-Ideals in Banach Spaces and Banach Algebras, Lecture Notes in Math., vol. 1547, Springer-Verlag, Berlin-Heidelberg-New York-London-Paris-Tokyo-Hong Kong-Barcelona-Budapest, 1993. MR 94k:46022

[LT] J. LINDENSTRAUSS and L. TZAFRIRI, Classical Banach Spaces I - Sequence Spaces, Springer-Verlag, Berlin-Heidelberg-New York, 1977. MR 58:17766

[R] C. E. RICKART, General Theory of Banach Algebras, D. Van Nostrand Company, Inc., Princeton, New Jersey-Toronto-London-New York, 1960. MR 22:5903

[TL] A. E. TAYLOR and D. C. LAY, Introduction to Functional Analysis (2nd edn), John Wiley \& Sons, New York-Chichester-Brisbane-Toronto, 1980. MR 81b:46001

Dipartimento di Matematica, Università di Genova, Via Dodecaneso 35, 16146 Genova, ITALY

E-mail address: burlando@dima.unige.it 